\title{
A New Method of Multi-criteria Group Decision Making Based on Hesitant-valued Intuitionistic Fuzzy and Osculating Value
}

\author{
Xiong Xianming ${ }^{1}$ \\ Business School of Sichuan University \\ Chengdu 610064, P. R. China \\ simon_panda@163.com \\ $\mathrm{Lu} \mathrm{Yi}{ }^{2, *}$ \\ Business School of Sichuan University \\ Chengdu 610064, P. R. China \\ luyiscu@163.com \\ * Corresponding Author
}

\author{
Muhammad Nazam ${ }^{3}$ \\ Business School of Sichuan University \\ Chengdu 610064, P. R. China \\ nazim_ehsas@yahoo.com
}

\begin{abstract}
This paper integrates the benefits of a intuitionistic fuzzy set (IFS) and a hesitant fuzzy set (HFS) to propose a new hesitant-valued intuitionistic fuzzy set (HVIFS). It retains the advantage of exchanging information in flexibility and exquisiteness, and also depicts the aspects of decision makers' hesitation in the membership degree and uncertainty. Hesitant-valued intuitionistic fuzzy (HVIF) value is able to better match the thinking mode and the real situation of decision makers (i.e. expert group). Considering the HVIFS numerical size depends on the size and stability, combine both of them to put forward a new scoring function. The Osculating Value method is able to reflect that whether the alternatives are close to the positive ideal scheme and away from the negative ideal scheme or not. Moreover, compared with VIKOR and TOPSIS methods, the Osculating Value use information more effectively and allows for a more accurate decision- making. According to these statements, a new approach of multi-attribute group decision making (MAGDM) based on HVIF and Osculating Value is proposed in this paper. In order to illustrate the effectiveness and efficacy of the method, apply it to evaluate a natural disaster emergency logistics scheme and the results showed that it is quite useful.
\end{abstract}

Keywords- Hesitant-valued intuitionistic fuzzy; Multiple attribute group decision making; Scoring function; Osculating Value; Emergency logistics

\section{INTRODUCTION}

Multi-attribute group decision making (MAGDM) is the combination of management science and decision science. As the social environment is becoming complex, more factors are needed to be taken into account in a decision-making process. Under these circumstances, MAGDM is attracting more attention in much research, which has been widely used in many fields, such as economic management, medical diagnosis.

Due to the complexity and uncertainty of practical objects and the limited knowledge of decision makers, the practical decision problems are always of great uncertainty. Zadch proposed fuzzy sets theory in 1965 [1], and then many researchers used it to handle uncertainties in various fields. In 1986, Atanassov extended the fuzzy sets theory and put forward intuitionistic fuzzy sets (IFS), which is able to make full use of the information in membership, non-membership and uncertainty. The proposal of IFS caused much attention. For example, Boran used IFSbased TOPSIS method to select appropriate suppliers [3]. Hwang applied the IFS to a pattern recognition problem [4]. De and Biswas used IFS method in a medical diagnosis decision [5]. However, it is deemed to be difficult to use precise values of non-membership and membership degree to depict IFS in some practical situations. To solve this issue, Atanassov and Gargov proposed an interval intuitionistic fuzzy set (IIFS) in 1989, which used interval-values to denote the membership degree, non-membership degree and uncertainty [6].

Due to the complexity in human minds and the differences of personal quality, decision makers often have different opinions and it is hard to agree on the same problem. For example, when decision-making panelists discuss the membership degree of one variable belonging to a particular set, some decision makers give 0.6 , some give 0.6 , and some give 0.8 . The panel members are independent individuals, so it is difficult to persuade each other. In order to deal with this complicated problem, Torra and Narukawa extended the fuzzy sets to the hesitant fuzzy sets (HFS), which uses a set of precise values to depict the membership degree [7, 8]. HFS allows that an element belonging to a set of membership is able to take several possible values. In this way, it can take into account preferences and views from different decision makers. In addition, HFS is able to handle the situation in which a decision maker is hesitant to choose from a couple of possible values [8]. For example, the qualitative concept of "twenty years old" can be represented as $\langle 19,0.85\rangle$ when using a fuzzy value, which means that the degree of nineteen years old belonging to twenty years old is 0.85 . Instead, it can be represented as $\{18,19,20\}$ when using a hesitant fuzzy number. Obviously, the latter method is able to better match the human brain thinking. 
As for the scoring function of a fuzzy number, Chen and Tan provided a scoring function of IFS in formula (1) [9]. Liu and Wang improved it and put forward formula (2), which is able to reflect the hesitation degree directly [10]. The formula (2) is composed of both the membership degree and the hesitation degree which represents the size of risk and uncertainty. From the geometric meaning of IFS, Szmidt and Kacprzyk put forward formula (3) [11]. It did not only consider the known information (i.e. membership degree and non-membership degree), which is measured by the distance of the object to the positive ideal point, but also the effect of the missing information, which is known as the degree of hesitation. If $D(b, a)$ represents the Hamming distance between $b$ and $a$ points, then formula (3) can be converted into formula (4). Compared with formula (1) and formula (2), formula (4) has stronger objectivity, and is able to consider more factors comprehensively.

(1)

$$
S(a)=\mu_{a}-v_{a} \quad\left(a=<\mu_{a}, v_{a}>, \mu_{a}+v_{a} \leq 1\right)
$$

$$
S(a)=\mu_{a}+\frac{\alpha_{\mu}}{\alpha_{\mu}+\beta_{\mu}} \pi_{a} \quad\left(\alpha_{\mu}+\beta_{\mu} \leq 1 \quad \alpha_{\mu}, \beta_{\mu} \in[0,1]\right)
$$

(2)

$$
\begin{aligned}
& S(a)=\frac{1}{2}\left(1+\pi_{a}\right) D(b, a) \\
& S(a)=\frac{1}{4}\left(1+\pi_{a}\right)\left(1-\mu_{a}+v_{a}+\pi_{a}\right)=\frac{1}{2}\left(1+\pi_{a}\right)\left(1-\mu_{a}\right)
\end{aligned}
$$

(4)

By integrating the advantages of IFS and HFS, reveal HVIFS, which is able to retain the advantage of IFS in fuzzy flexibility and reflect decision makers' hesitation in membership degree, non-membership degree and uncertainty. Considering the value of HVIFS depends on the size of the scoring function in formula (5) [12] and the stability of the scoring function in formula (6), this paper combines the two formulas for a better score function in formula (7) for HVIFS. Since the Osculating Value is able to reflect whether the alternatives are close to the positive ideal point and away from the negative ideal point or not, the Osculating Value method can use data information more effectively and make decisions more accurately compared to VIKOR and TOPSIS methods. This paper puts forward a new method of MAGDM based on HVIF and Osculating Value. It is able to better match the thinking mode and the real situation of human decision makers (i.e. expert group). Moreover, the involvement of the non-membership degree is able to describe the fuzziness of things more exquisitely. In order to illustrate the effectiveness and efficacy of the method, apply it to the evaluation of natural disaster emergency logistics schemes.

The remainder of this paper is structured as follows: Section 2 defines related concepts, such as FVIFS and the scoring function; Section 3 combines the Osculating Value method to construct a new method of MAGDM; Section 4 applies this method to evaluate natural disaster emergency logistics schemes. Section 5 concludes this paper with a summary.

\section{PRELIMINARIES}

Definition 1 [8]: For $\forall x \in X$, if $\forall u_{a}(x) \in \Gamma_{A}(x)$, $\exists v_{a}(x) \in \Psi_{A}(x), \quad 0 \leq u_{a}(x)+v_{a}(x) \leq 1$, then the set $A=\left\{<x, \Gamma_{A}(x), \Psi_{A}(x)>\mid x \in X\right\}$ is called a HVIFS. For $x_{j} \in X$, the hesitation degree set of $A$ is $\Pi_{A}\left(x_{j}\right)$ $=\bigcup\left\{1-u_{a}(x)-v_{a}(x) \mid 1-u_{a}(x)-v_{a}(x) \geq 0\right\} . \quad \Pi_{A}\left(x_{j}\right)$

measures the uncertainty of $x_{j}$ belonging to $A$, where $\pi_{a}\left(\mathrm{x}_{j}\right)=1-u_{a}(x)-v_{a}(x), 0 \leq \pi_{a}\left(\mathrm{x}_{j}\right) \leq 1$. Specifically, if $\forall x \in X, \Gamma(x)$ and $\Psi(x)$ only contain one element, the HVIFS degrades into a IFS; if $\forall x \in X, \Psi(x)=\{0\}$, the HVIFS degrades into a HFS; if the $X$ set contains only one element, then $\left\langle x, \Gamma_{A}(x), \Psi_{A}(x)\right\rangle$ is called as hesitant-valued intuitionistic fuzzy number (HVIFN), marked as $<\Gamma_{A}, \Psi_{A}>$.

Definition 2 [8]: In the collection of $X$, let $A=<\Gamma_{A}$, $\Psi_{A}>\in \operatorname{HVIFN}(X)\left(\mu_{a} \in \Gamma_{A}, v_{a} \in \Psi_{A} \mu_{a}+v_{a} \leq 1\right)$, then the score function of $A$ is defined as formula (5), Where $s(a)$ is showed as is in formula (4), \#A is the number of elements in the collection $A$. The value of $r(A)$ is greater, the HVIFS of $A$ is smaller.

$$
r(A)=\frac{1}{\# A} \sum_{a \in A} s(a)
$$

Definition 3 [12]: In the collection of $X$, let $A=<\Gamma_{A}$, $\Psi_{A}>\in \operatorname{HVIFN}(X)\left(\mu_{a} \in \Gamma_{A}, v_{a} \in \Psi_{A} \mu_{a}+v_{a} \leq 1\right)$, then the uncertain function of $u(A)$ is defined as formula (6). The value of $u(A)$ shows the uncertainty $A$ is. The greater the $u(A)$ value, the smaller $A$ is.

(6)

$$
u(A)=\frac{1}{\# A} \sum_{A \in A}(s(a)-r(A))^{2}
$$

Definition 4: In the collection of $X$, let $A=<\Gamma_{A}$, $\Psi_{A}>\in \operatorname{HVIFN}(X)$, then the scoring function of $A$ is defined as formula (7), where $10^{4}$ is to reduce the order of magnitude and avoid excessive value. When $f(A)$ is greater, the HVIFS of $A$ is greater.

(7)

$$
f(A)=\frac{1}{10^{4} r(A) * u(A)}
$$

\section{A NEW METHOD OF MCGDM BASED ON HVIFS AND OSCULATING VALUE}

In this paper, the method of MCGDM integrates the HVIFS and the Osculating Value. Specific decision making steps are shown as follows:

Step 1 Build a multiple attribute decision making matrix. 
For a multiple attribute decision making problem, if $A_{1}, A_{2}, \cdots, A_{m}$ is $m$ evaluation schemes, then $C=\left\{c_{1}\right.$, $\left.c_{2}, \cdots, c_{m}\right\}$ is a set of property, $h_{i j}$ is the value of HVIF which is given by several experts under the scheme of $A_{i}$ and the property of ${ }^{c_{j}}$. Therefore, get a matrix $H$ of HVIF, where $h_{i j}=<\Gamma_{A}\left(x_{j}\right), \Pi_{A}\left(x_{j}\right), \Psi_{A}\left(x_{j}\right)>, \Gamma_{A}\left(x_{j}\right)$ means the collection of membership degree of $x_{j}$ belonging to $A, \Psi_{A}\left(x_{j}\right)$ means the collection of nonmembership degree, $\Pi_{A}\left(x_{j}\right)$ means the collection of uncertainty.

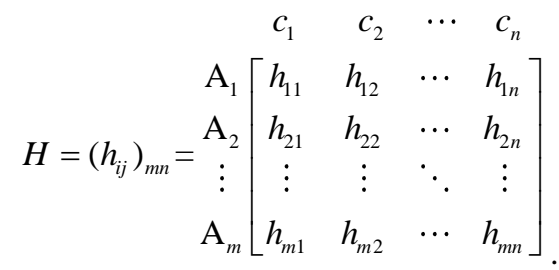

Step 2 Standardize the decision matrix.

For the scheme $A_{i}$, the element number of membership degree set, hesitation degree set and non-membership degree set in $h_{i j}$ may be different from other schemes under the same property of $x_{j}$. In order to get the standardized decision matrix, the method from literature [13] is applied. In the $k$ th row of matrix $H$, let the number of membership degree set, uncertainty set and nonmembership degree set correspond to the same number among $h_{1 k}, h_{2 k}, \cdots, h_{m k}$. Moreover, the elements in these sets are ordered from the biggest to the smallest in accordance with the values. For example, $l=\max \left\{\# \Gamma_{1}\right.$, $\left.\# \Gamma_{2}, \cdots, \# \Gamma_{k}\right\}, \# \Gamma$ means the number of elements from set $\Gamma$, if the values of $\# \Gamma_{1}, \# \Gamma_{2}, \cdots, \# \Gamma_{k}$ are not all equal to each other, then add elements to the set whose element number is less than $l$ until the values of $\# \Gamma_{1}, \# \Gamma_{2}, \cdots, \# \Gamma_{k}$ are the same. Under the pessimistic principle, the added element is the smallest element. However, under the rule of optimistic, the added element is

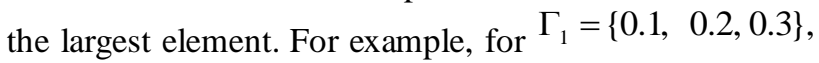
$\Gamma_{2}=\{0.4,0.5\}$ there is a need to add element to $\Gamma_{2}$ until $\Gamma_{1}$ and $\Gamma_{2}$ have the same number of elements. Therefore, under the pessimistic principle, $\Gamma_{2}=\{0.4,0.4,0.5\}$

Step 3 Build a decision-making score matrix.

Calculate the values of $r(A), u(A)$ and $f(A)$, then HVIFS of $h_{i j}$ can be converted to a score value of $f_{i j}$, the decision matrix $H$ can be converted into score matrix $F$,

$$
\begin{aligned}
& \begin{array}{cccc}
c_{1} & c_{2} & \cdots & c_{n}
\end{array} \\
& F=\left(f_{i j}\right)_{m n}=\begin{array}{c}
\mathrm{A}_{1} \\
\mathrm{~A}_{2} \\
\vdots \\
\mathrm{A}_{m}
\end{array}\left[\begin{array}{cccc}
f_{11} & f_{12} & \cdots & f_{1 n} \\
f_{21} & f_{22} & \cdots & f_{2 n} \\
\vdots & \vdots & \ddots & \vdots \\
f_{m 1} & f_{m 2} & \cdots & f_{m n}
\end{array}\right] .
\end{aligned}
$$

Step 4 Find out the positive and negative ideal schemes. Positive ideal scheme is defined as $A^{+}=\left\{f_{1}^{+}, f_{2}^{+}\right.$, $\left.\cdots, f_{n}^{+}\right\}(j=1,2, \cdots, n)$, negative ideal scheme is defined as $A^{-}=\left\{f_{1}^{-}, f_{2}^{-}, \cdots, f_{m}^{-}\right\}$,

$$
\begin{gathered}
\left(f_{j}\right)^{+}=\left\{\begin{array}{ll}
\max _{i=1, \cdots, m}\left\{f_{j}\right\} & j \in B_{T} \\
\min _{i=1, \cdots, m}\left\{f_{j}\right\} & j \in C_{T}
\end{array},\right. \\
\left(f_{j}\right)^{-}=\left\{\begin{array}{ll}
\min _{i=1, \cdots, m}\left\{f_{j}\right\} & j \in B_{T} \\
\max _{i=1, \cdots, m}\left\{f_{j}\right\} & j \in C_{T}
\end{array},\right.
\end{gathered}
$$

for $B_{T}$ is the quality-benefit type attribute set, $C_{T}$ is the cost type attribute set.

Step 5 Calculate the weighted distance.

If the corresponding attribute weight vector is $w=\left(w_{1}, w_{2}, \cdots, w_{n}\right), \quad \sum_{j=1}^{n} w_{j}=1, \quad$ than calculate the weighted distance of $A_{i}$ to $A^{+}$and $A^{-}$, shown as $d_{i}^{+}=\sqrt{\sum_{j=1}^{n} w_{j}\left(f_{i j}-f_{j}^{+}\right)^{2}}, d_{i}^{-}=\sqrt{\sum_{j=1}^{n} w_{j}\left(f_{i j}-f_{j}^{-}\right)^{2}}$

respectively.

Step 6 Calculate the Osculating Value of each alternative.

Osculating Value $\left(C_{i}\right)$ reflects the extent of alternatives being close to the positive ideal scheme and being from negative ideal scheme. It is denoted as $C_{i}=\frac{d_{i}^{+}}{d^{+}}-\frac{d_{i}^{-}}{d^{-}}, i=1,2, \cdots, m$

Where $d^{+}$means the minimum distance of schemes to the positive ideal scheme; $d^{-}$means the maximum distance of schemes to the negative ideal scheme, namely $d^{+}=\min _{1 \leq i \leq m}\left\{d_{i}^{+}\right\}$, $d^{-}=\max _{1 \leq i \leq m}\left\{d_{i}^{-}\right\}$

Step 7 Order all of the schemes from the biggest to the smallest according to the size of the Osculating Value $C_{i}$. The smaller the Osculating Value, the better the scheme.

\section{CASE STUDY}

Emergency logistics is of vital significance in natural disaster rescue. In the process of emergency logistics scheme selection, it is necessary to take into account multiple factors. Under the complicated environment, decision making often need many experts, rather than one or two individuals. Since emergency logistics often take places in information block, pressed for time and traffic jam, the environment of decision making is much more complicated than traditional logistics, especially under the 
natural disaster. Under these circumstances, experts' evaluation has a great deal of uncertainty, fuzziness is one of the major uncertainties. When choosing optimal emergency logistics scheme, the experts need to consider many factors, such as time, cost and safety.

The evaluation of emergency logistics scheme is a MAGDM problem. This paper applies the method mentioned above to evaluate emergency logistics schemes from the example of [12]. In a natural disaster relief activity, the experts provided three alternative emergency

$$
w=(0.202,0.225,0.137,0.107,0.183,0.146)^{T} .
$$

logistics schemes: $A_{1}, A_{2}, A_{3}$, each one under the following six principles: (1) $c_{1}$ is the easiness to get emergency supplies; (2) $c_{2}$ is the timeliness to transport emergency supplies; (3) $c_{3}$ is the undamaged ratio to pipe emergency supplies; (4) $c_{4}$ is the safety of the transportation network; (5) $c_{5}$ is the material cost; (6) $c_{6}$ is the transportation cost. The weight of these attributes is

The expert panels evaluate $A_{1}, A_{2}, A_{3}$ under these six properties when evaluation information is given in the form of HVIFS, as shown in TABLE I.

TABLE I. EVALUATION INFORMATION OF SCHEMES

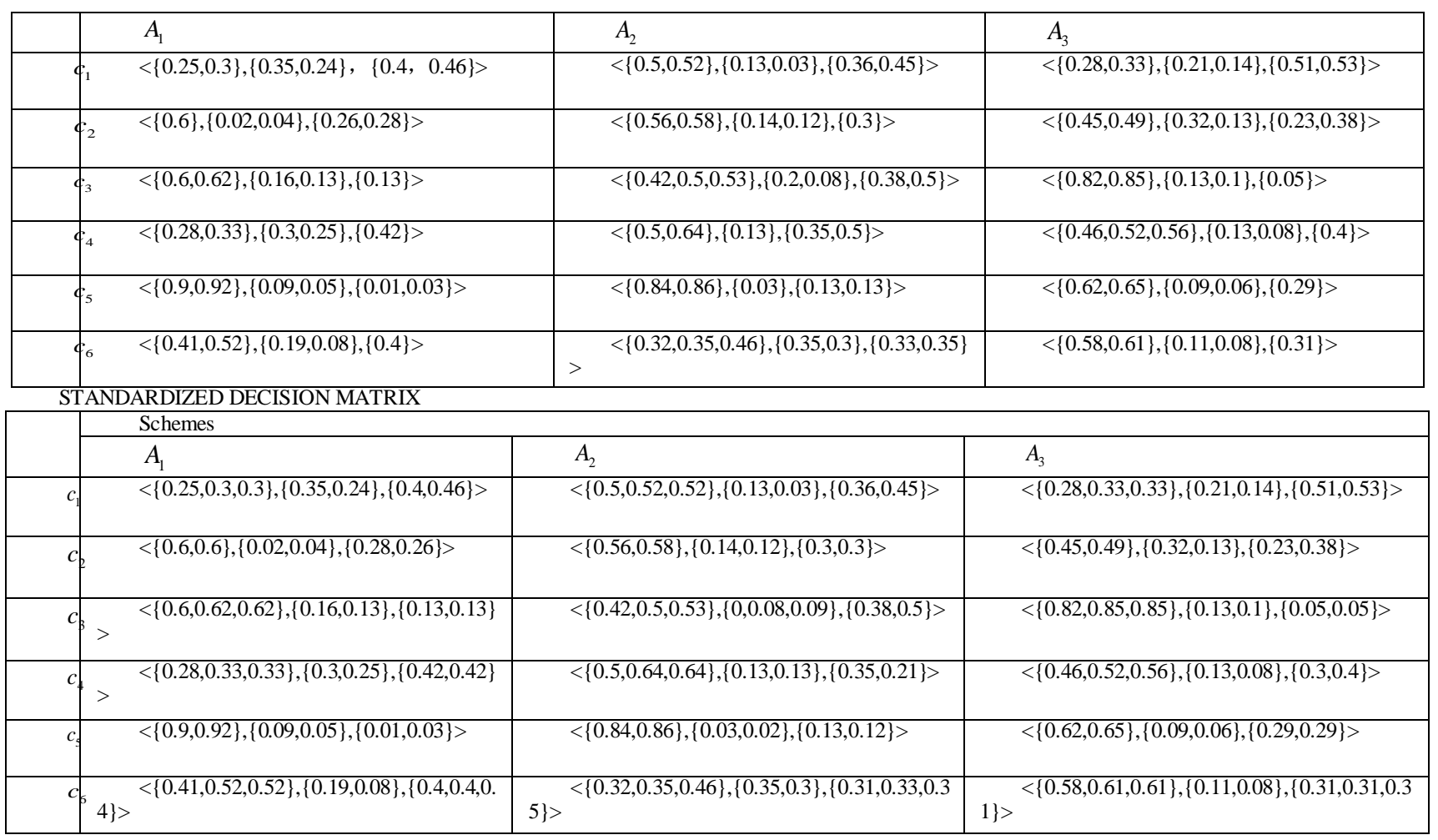

Step 1 Construct a multiple attribute decision making matrix. Under the attributes of $c_{1}, c_{2}, \cdots, c_{6}$, the experts make evaluation of the $A_{1}, A_{2}, A_{3}$ schemes, which are based on the HVIF. As a result, build a multi-criteria decision making matrix based on HVIF.

Step 2 Standardize the decision matrix. In this paper, use the optimistic principle standardized membership degree sets, and the pessimistic principle standarized hesitant degree sets and the non-membership degree sets. After that, the standardized decision matrix is determined, as shown in TABLE TABLE 2.

Step 3 Build a decision-making score matrix. By calculating the scoring function of HVIFS, the HVIFN of $h_{i j}$ is converted into a scoring value $f_{i j}$, then the matrix of $H^{\prime}$ is converted into to score matrix $F$, shown as follows:

$$
\begin{aligned}
& \begin{array}{cccccc}
c_{1} & c_{2} & c_{3} & c_{4} & c_{5} & c_{6}
\end{array} \\
& F=\left(f_{i j}\right)_{m n}=\begin{array}{l}
A_{1} \\
A_{2} \\
A_{3}
\end{array}\left[\begin{array}{cccccc}
0.16 & 0.21 & 11.31 & 0.37 & 53.06 & 0.16 \\
1.21 & 13.53 & 0.17 & 0.25 & 42.99 & 0.09 \\
0.34 & 13.53 & 11.78 & 0.66 & 4.37 & 3.56
\end{array}\right] \text {. }
\end{aligned}
$$

Step 4 Find out the positive and negative ideal schemes. The ideal scheme and negative scheme are shown as $A^{+}=\{1.21,13.53,11.78,0.66,4.37,0.09\}$;

$A^{-}=\{0.16,0.21,0.17,0.25,53.06,3.56\}$

Step 5 Calculate the weighted distance. $w=(0.202$, $0.225,0.137,0.107,0.183,0.146)^{T}$ is the attribute weight vector, so the weighted distance of $A_{i}(i=1,2,3)$ to $A^{+}$and $A^{-}$can be calculated, which are shown as: $d_{1}^{+}=21.77, \quad d_{1}^{-}=14.12 ; \quad d_{2}^{+}=17.07, \quad d_{2}^{-}=7.78 ;$ $d_{3}^{+}=13.38, d_{3}^{-}=12.19$. 
Step 6 Calculate the Osculating Value of the three schemes. Before calculating the Osculating Value, it is needed to determine the values of $d^{+}, d^{-}$, where $d^{+}$represents the minimum distance of the schemes to $A^{+}$ $d^{-}$represents the maximum distance of the schemes to $A^{-}$, namely $d^{+}=\min _{1 \leq i \leq m}\left\{d_{i}^{+}\right\}=13.38, \quad d^{-}=\max _{1 \leq i \leq m}\left\{d_{i}^{-}\right\}$ $=14.12$. Then calculate the Osculating Value of three schemes, shown as: $C_{1}=0.627, C_{2}=0.725, C_{3}=0.137$ respectively.

Step 7 According to Osculating Values, the descending order of three schemes is written as below $C_{2} \succ C_{1} \succ C_{3}$. This means $C_{2}$ is the optimal scheme.

\section{SUMMARY}

In current MAGDM research, only a few researchers have focused on using HVIFS as an evaluation value of attribute. To narrow the literature gap and promote the importance of hesitation in decision making, propose a modified method for MAGDM. The proposed approach is based on HVIFS and combines the Osculating Value. In addition, this paper put forward a new definition of the score function of HVIFS, which is able to convert HVIFS into comparison values. From the application example to emergency logistics scheme selection, the method is shown to be feasible and effective. This method also reflects the extent of alternatives being close to the positive ideal scheme and being away from negative ideal scheme. It has theoretically value and practically significant to some extent. It can be widely used in other fields, such as choosing suppliers, purchasing goods, and project evaluation.

The main limitation of developed approach is related to that the experts participated in this research are limited. Since the method used in this paper relies on knowledge and experience of experts, it is vital to use elite experts. Future research may focus on developing new emergency logistics risk indexes by incorporating the new frameworks, such as the various artificial intelligence modeling techniques, multi-agent, graph theory, gray theory, and game theory.

\section{ACKNOWLEDGMENT}

This research is supported by China Postdoctoral Science Foundation (Grant No. 2014M550470). The authors are also grateful to Sichuan University for providing research funding, No. SKG2013001.

\section{REFERENCES}

[1] A. Zadeh, Fuzzy sets [J]. Information and Control, 1965, 8(3):338 353.

[2] K. Atanassov, Intuitionistic fuzzy sets [J]. Fuzzy Sets and Systems, 1986, 20(1):86 96.

[3] F. E. Boran, S. Genc, M. Kurt, D. Akay, A multi-criteria intuitionistic fuzzy group decision making for supplier Selection with TOPSIS method [J]. Expert Systems with Applications, 2009, 36:11363-11368.

[4] C. M. Hwang, M. S. Yang, W. L. Hung, M. G. Lee, A similarity measure of intuitionistic fuzzy sets based on the sugeno integral with its application to pattern recognition [J]. Information Sciences, 2012, 189: 93-109.

[5] S. K. De, R. Biswas, A. R. Roy, An application of intuitionistic fuzzy sets in medical diagnosis [J]. Fuzzy Sets and Systems, 2001, 116(2):209-213.

[6] K. Atanassov, G. Gargov, Inteval-valued intuitionistic fuzzy sets [J]. Fuzzy Sets and Systems, 1989, 31(1):343-349.

[7] V. Torra, Y. Narukawa, On hesitant fuzzy sets and decision [C] // Fuzzy Systems, the 18th IEEE International Conference, 2009:1368-1382.

[8] V. Torra, Hesitant fuzzy sets [J]. International Journal of Intelligent Systems, 2010, 25(6):529-539.

[9] S. M. Chen, J. M. Tan, Handling multi-criteria fuzzy decision making problems based on vague set theory [J]. Fuzzy Sets and Systems, 1994, 66(2):163-162.

[10] H. W. Liu, G. J. Wang, Multi-criteria decision-making methods based on intuionistic fuzzy sets [J]. European Journal of Operational Research, 12006, 69:220-233.

[11] E. Szmidt, J. Kacprzyk, Ranking of intuitionistic fuzzy alternatives in a multi-criteria decision making problem [C]. The 28th North American Fuzzy Information Processing Society Annual Conference (NAFIPS2009) Cincinnati, Ohio, USA, 2009, 6:14-16.

[12] P. Lu, The study of intuitionistic hesitant fuzzy multi-criteria group decision-making methods [D]. Central South University. 2013, $11: 34$.

[13] N. Zhang, G W. Wei, Extension of VIKOR method for decision making problem based on hesitant fuzzy set [J]. Applied Mathematical Modeling. 2013, 36(6):4938-4946. 